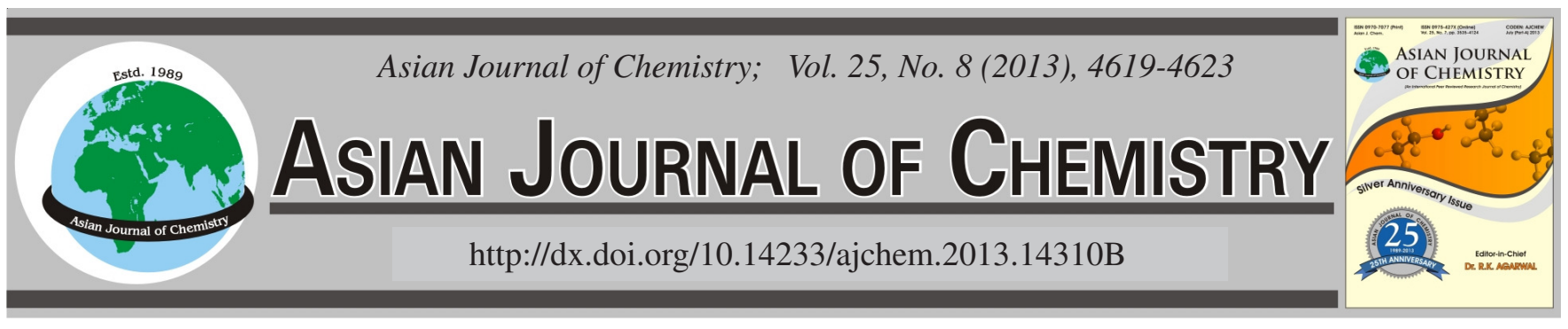

\title{
Drug Release Study from Superabsorbent Hydrogel Based on Poly-2-acrylamido-2-methylpropanesulfonic Acid Grafted Collagen
}

Fatemeh Soleimani ${ }^{*}$, Hossein Sadeghi, Hadis Shahsavari, Arezou Soleimani and Fatemeh Sadeghi

Department of Chemistry, Science Faculty, Arak Branch, Islamic Azad University, Arak, Iran

*Corresponding author: E-mail: fatisoleymani@yahoo.com

(Received: 13 July 2012;

Accepted: 18 February 2013)

AJC-13026

The present work focused on the design of drug delivery system based on a pH-sensitive hydrogel. The hydrogels were prepared via graft copolymerization of 2-acrylamido-2-methylpropane sulfonic acid monomer onto collagen backbones by a free radical polymerization technique. Sodium bicarbonate was added to function as a foaming agent under acidic conditions, rendering the hydrogels to be porous. Porous structure of hydrogel was essential in this system to yield a large surface area so that acetaminophen release could be facilitated. The hydrogel thus prepared possessed a porous structure as determined by scanning electron microscopy. Due to the reversible swelling behaviour of the hydrogels, the synthesized networks can sense the environmental $\mathrm{pH}$ change and achieve an oscillatory release pattern. The concentration of released acetaminophen loaded was monitored at $245 \mathrm{~nm}$ on the UV spectrophotometer. Water absorption of the hydrogel could be switched on and off swiftly by control of $\mathrm{pH}$ of the surrounding environment. Therefore, the synthesized hydrogels in this work can be used as a drug delivery system and that the drug release can be controlled by the $\mathrm{pH}$ of solution.

Key Words: Collagen, Hydrogel, 2-Acrylamido-2-methylpropanesulfonic acid, Acetaminophen, Releasing drug.

ᄂ - - - - - - - - - - - - - - - - - - - - - - - - - -

\section{INTRODUCTION}

The market for superabsorbent polymers (SAPs) has increased by a factor of 5 over the past 10 years. These materials are crosslinked hydrophilic polymers, capable of absorbing large quantities of water, saline or physiological solutions $s^{1,2}$. The absorbed fluids are hardly removable even under some pressure. They are widely used in various applications such as hygienic, foods, cosmetics and agriculture ${ }^{2-4}$. This accounts for increase in the worldwide production of superabsorbent polymers from 6000 tons in 1983 to 450000 tons in $1996^{1}$. Nowadays, the worldwide production of superabsorbent polymers is more than one million tons in year. Hence, synthesis and investigation of specific and new superabsorbent hydrogels with high absorbency, mechanical strength and initial absorption rate, is the main goal of the several research groups in the world ${ }^{5-9}$.

The properties of the swelling medium (e.g., $\mathrm{pH}$, ionic strength and the counter ion and its valency) affect the swelling characteristics. Superabsorbent polymers responding to external stimuli such as heat, $\mathrm{pH}$, electric field, chemical environments, etc., are often referred to as "intelligent" or "smart" polymers. Among these, $\mathrm{pH}$-sensitive hydrogels have been extensively investigated for potential use in site-specific delivery of drugs to specific regions of the gastrointestinal tract and have been prepared for delivery of low molecular weight protein drugs. Therefore, these hydrogels have important applications in the field of medicine, pharmacy and biotechnology ${ }^{10,11}$.

Drug release from solid matrices systems, made of polymer(s) and $\operatorname{drug}(\mathrm{s})$, is a basic concept for studies on controlled drug delivery. The most interesting class of polymers in this application is given by hydrogels, also $\mathrm{pH}$-sensitive ones. Hydrogels are special soft and pliable polymeric materials that can be absorb large quantities of water, saline or physiological solutions while the absorbed solutions are not removable even under pressure. In the swollen state, these become soft and rubbery, resembling a living tissue and some possess excellent biocompatibility ${ }^{3}$. Thus, polymeric hydrogels are of considerable interest as biomaterials in drug delivery research.

In the current study we investigated the synthesis and utility of an anionic hydrogel from graft copolymerization of 2-acrylamido-2-methylpropane sulfonic acid (AMPS) monomer onto collagen backbones for the controlled release of a model drug, acetaminophen. Drug absorption and release capacities of hydrogel systems and influence of $\mathrm{pH}$ of the medium, porosity and the crosslinker content on the release properties were also examined. 


\section{EXPERIMENTAL}

Hydrolyzed collagen (Parvar Novin-E Tehran Co.) was industrial grade which is available in market and has nearly $25 \%$ insoluble phosphate salt. 2-Acrylamido-2-methylpropane sulfonic acid (AMPS) (Merck, Darmstadt, Germany), N',N'methylene bisacrylamide and ammonium persulphate (Fluka, Buchs, Switzerland) were of analytical grade and used without further purification. The drug, acetaminophen, was obtained from Jaberebne Hayan Pharmaceutical Co. (Tehran, Iran). The chemical structure of drug is shown in Fig. 1. Double distilled water was also used for the hydrogel preparation and swelling measurements.

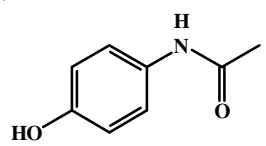

Fig. 1. Chemical structure of drug acetaminophen

Preparation of hydrogel: A pre-weighed amount of hydrolyzed collagen (1.0-4.0 g) was dissolved in $40 \mathrm{~mL}$ degassed distilled water and filtered to remove its insoluble salt. The solution was added to a $1 \mathrm{~L}$ three-neck reactor equipped with a mechanical stirrer (RZR 2021, a three-blade propeller type, Heidolph, Schwabach, Germany) and the reactor was immersed in a thermostated water bath preset at a desired temperature $\left(65^{\circ} \mathrm{C}\right)$. Then 2-acrylamido-2-methylpropane sulfonic acid (2.0-8.0 g) was added to the reactor. After stirring for $10 \mathrm{~min}$, ammonium persulphate (0.01-0.40 g APS in $5 \mathrm{~mL}$ $\left.\mathrm{H}_{2} \mathrm{O}\right)$ and methylene bisacrylamide $\left(0.05-0.20 \mathrm{~g}\right.$ in $\left.5 \mathrm{~mL} \mathrm{H}_{2} \mathrm{O}\right)$ were added simultaneously to the reaction mixture. The temperature was maintained at $65^{\circ} \mathrm{C}$ and the reaction mixture was stirred continuously ( $300 \mathrm{rpm}$ ) for $1 \mathrm{~h}$. At the end of the propagation reaction, the gel product was poured into ethanol (200 $\mathrm{mL}$ ) and was dewatered for $12 \mathrm{~h}$. Then, the product was cut into small pieces, washed with $200 \mathrm{~mL}$ ethanol and filtered. The particles were dried in an oven at $50{ }^{\circ} \mathrm{C}$ for $12 \mathrm{~h}$. After grinding, the powdered superabsorbent hydrogel was stored in absence of moisture, heat and light.

Swelling measurements: An accurately weighed sample $(0.2 \pm 0.001 \mathrm{~g})$ of the powdered superabsorbent with average particle sizes between 40-60 mesh (250-350) was immersed in distilled water $(200 \mathrm{~mL})$ and allowed to soak for $3 \mathrm{~h}$ at room temperature. The equilibrium swelling (ES) capacity was measured twice at room temperature according to a conventional tea bag (i.e., a 100 mesh nylon screen) method and using the following formula:

$$
\text { ES }(\mathrm{g} / \mathrm{g})=\frac{\text { Weight of swollen gel }- \text { Weight of dried gel }}{\text { Weight of dried gel }}
$$

Instrumental analysis: Fourier transform infrared (FTIR) spectroscopy absorption spectra of samples were taken in $\mathrm{KBr}$ pellets, using an ABB Bomem MB-100 FTIR spectrophotometer (Quebec, Canada), at room temperature. To study the morphology of the hydrogel, the surface and cross-sectioned area of the hydrogel were examined using scanning electron microscopy (SEM). After Soxhlet extraction with methanol for $24 \mathrm{~h}$ and drying in an oven, superabsorbent powder was coated with a thin layer of gold and imaged in a SEM instrument
(Leo, 1455 VP). Brunauer-Emmett-Teller (BET) analysis was used to determine the pore size of the hydrogels.

Determination of drug loading: Loading model drug into a hydrogel was performed using a contact adsorption technique. The vacuum dried powdered samples $(1 \pm 0.0001$ $\mathrm{g}$ ), with average particle sizes between 40 and 60 mesh (250$350 \mu \mathrm{m})$, were accurately weighted and immersed in the aqueous solution of drug $(0.6 \mathrm{~g}$ dissolved in $50 \mathrm{~mL}$ distilled water) at $0{ }^{\circ} \mathrm{C}$ for $25 \mathrm{~h}$ to reach the equilibrated state. The swollen hydrogels loaded with drug were placed in a vacuum oven and dried under vacuum at $37^{\circ} \mathrm{C}^{5}$.

The amount of drug content entrapped in the hydrogels was determined by an indirect method. After the gel preparation, the washings were collected, filtered with a $0.45 \mu \mathrm{m}$ Millipore filter and tested at $\lambda_{\max } 245 \mathrm{~nm}$ using UV/VIS spectrophotometer (UV-1201, Shimadzu, Kyoto, Japan).

The drug entrapped exhibited the same $\lambda_{\max }$ as the free drug. This clearly indicates that the drugs entrapped have not undergone any possible chemical reaction during the matrix formation. The difference between the amount of drug initially employed and the drug content in the washings is taken as an indication of the amount of drug entrapped ${ }^{6}$.

Drug entrapment (\%)

$$
=\frac{\text { Mass of drug present in hydrogel }}{\text { Theoretical mass of drug }} \times 100
$$

Standard absorbance curve: The standard calibration curve of the absorbance as a function of drug concentration was studied at $245 \mathrm{~nm}$ on the UV spectrophotometer.

In vitro drug release: The samples $(0.1 \pm 0.0001 \mathrm{~g})$ were immersed into $50 \mathrm{~mL}$ of the release medium [simulated gastric fluid (SGF) and simulated intestinal fluid (SIF)] with different $\mathrm{pH}$ values ( $\mathrm{pH} 1.2$ or 7.4 ) at $37^{\circ} \mathrm{C}$ with agitation. At given time intervals, $1 \mathrm{~mL}$ of the release medium was removed using a syringe attached with a $0.45 \mu \mathrm{m}$ Millipore filter and after suitable dilution the concentration of released drug was measured by UV spectrophotometer at $245 \mathrm{~nm}$. The drug release per cent was calculated twice using the following equation:

$$
\text { Released drug }(\%)=\frac{\mathrm{R}_{\mathrm{t}}}{\mathrm{L}} \times 100
$$

where $L$ and $R_{t}$ represent the initial amount of drug loaded and the final amount of drug released at time $t$.

\section{RESULTS AND DISCUSSION}

Synthesis and spectral characterization: The mechanism for crosslinking graft copolymerization of 2-acrylamido-2methylpropane sulfonic acid onto collagen backbones in the presence of ammonium persulfate and N',N'-methylene bisacrylamide is shown in Scheme-I. In the first step, the thermally dissociating initiator, i.e., ammonium persulfate, is decomposed under heating $\left(80{ }^{\circ} \mathrm{C}\right)$ to produce sulphate anion-radicals. Then, the anion-radicals abstract hydrogen from one of the functional groups (i.e., $\mathrm{COOH}, \mathrm{SH}, \mathrm{OH}$ and $\mathrm{NH}_{2}$ ) in side chains of the collagen backbones to form corresponding macro-initiators. These macroradicals initiate grafting of 2-acrylamido-2-methylpropane sulfonic acid onto collagen backbones leading to a graft copolymer. Crosslinking reaction also occurred in the presence of the crosslinker, i.e., N',N'methylene bisacrylamide. 


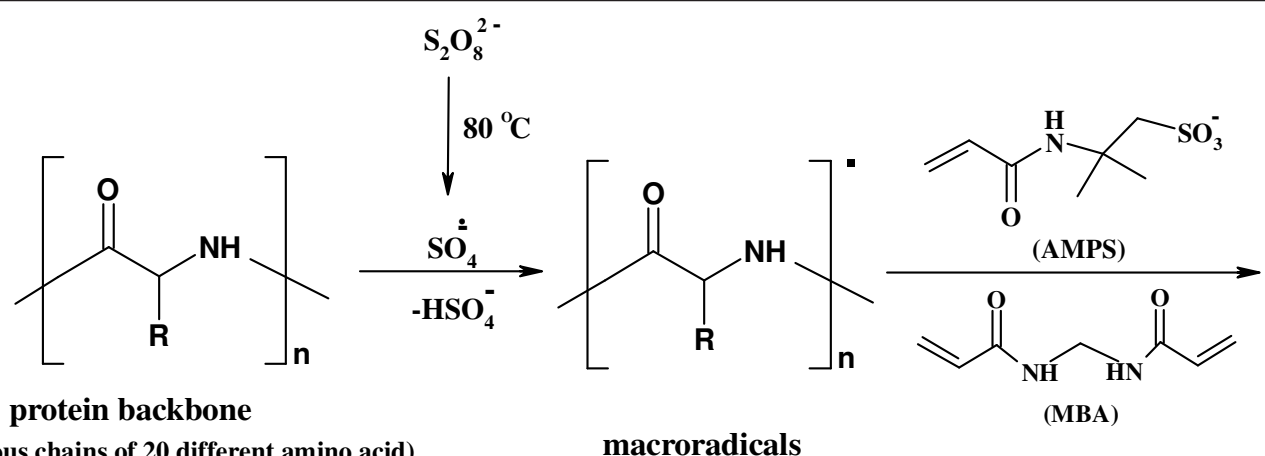

(R: various chains of $\mathbf{2 0}$ different amino acid)

macroradicals

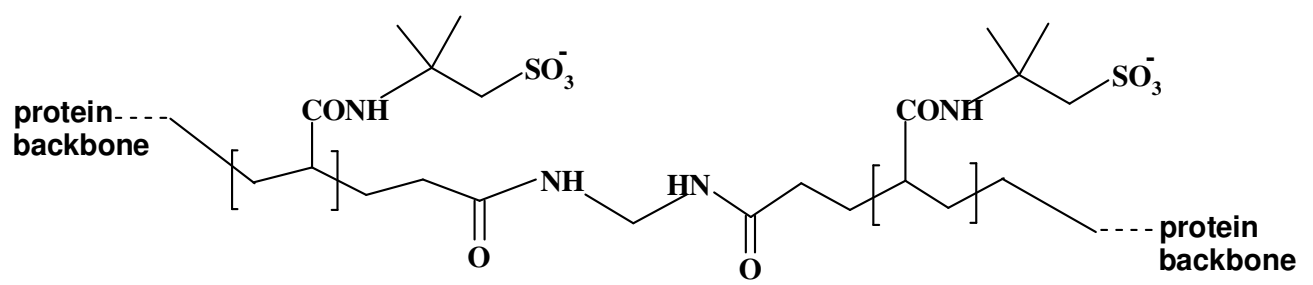

(crosslinked collagen-g-AMPS hydrogel)

Scheme-I: Proposed mechanistic pathway for synthesis of collagen-g-poly (AMPS) hydrogel

FTIR spectroscopy was used for identification of the hydrogel. Fig. 2 shows the IR spectra of the collagen and the resulted hydrogel. The band observed at $1658 \mathrm{~cm}^{-1}$ can be attributed to $\mathrm{C}=\mathrm{O}$ stretching in carboxamide functional groups of substrate backbone (Fig. 2a). The broad band at 3600-3200 $\mathrm{cm}^{-1}$ is due to stretching of -OH groups of the collagen. The collagen-g-AMPS hydrogel comprises a collagen backbone with side chains that carry sulphate groups that are evidenced by a new characteristic absorption band at $1221 \mathrm{~cm}^{-1}$ (Fig. 2b). This peak attributed to ester sulphate stretching of 2-acrylamido2-methylpropane sulfonic acid. The stretching band of - $\mathrm{NH}$ overlapped with the $\mathrm{OH}$ stretching band of the collagen portion of the copolymer.

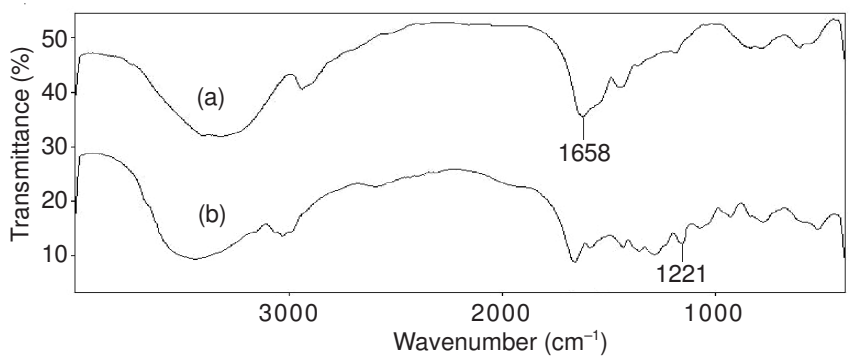

Fig. 2. FTIR spectra of collagen (a) and collagen-g-poly (AMPS) hydrogel (b)

To obtain additional evidence of grafting, a similar polymerization was conducted in the absence of the crosslinker. After extracting the homopolymer and unreacted monomers using a cellophane membrane dialysis bag (D9402, SigmaAldrich), an appreciable amount of grafted collagen (87\%) was observed. The graft copolymer spectrum was very similar to Fig. 2b. Also according to preliminary measurements, the sol (soluble) content of the hydrogel networks was as little as $1.8 \%$. This fact practically proves that all 2 -acrylamido- 2 methylpropane sulfonic acid are almost involved in the polymer network. So, the monomers percent in the network will be similar to that of the initial feed of reaction.
Scanning electron microscopy: One of the most important properties that must be considered is hydrogel microstructure morphologies. Fig. 3 shows the scanning electron microscope (SEM) photographs of the surface (Fig. 3a) and the crosssectional area (Fig. 3b) of the hydrogel with interconnected pores. These pictures verify that the synthesized polymer in this work have a porous structure, where the pores might be induced into the hydrogel by water evaporation resulting from reaction heat. It is supposed that these pores are the regions of water permeation and interaction sites of external stimuli with the hydrophilic groups of the graft copolymers. The crosssectional view of hydrogels (Fig. 3b) also exhibited large, open, channel-like structure.
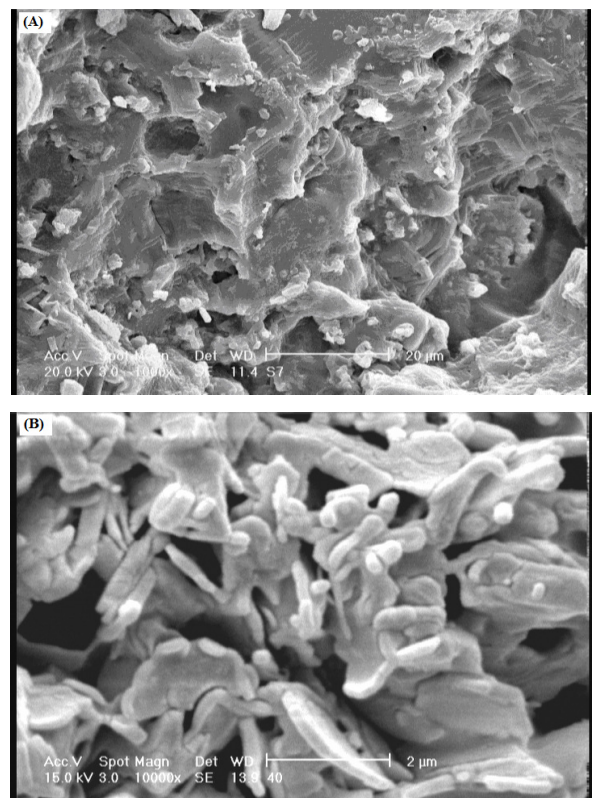

Fig. 3. SEM photograph of the optimized superabsorbent hydrogel (collagen $3.0 \mathrm{~g}$, MBA $0.008 \mathrm{~mol} / \mathrm{L}$, AMPS $0.68 \mathrm{~mol} / \mathrm{L}$, APS 0.015 $\mathrm{mol} / \mathrm{L}, 80^{\circ} \mathrm{C}, 1 \mathrm{~h}$ ). (A) Surface of porous hydrogel; (B) crosssectional area of porous hydrogel. The average pore diameter of the synthesized hydrogel was $16.7 \mathrm{~nm}$ 
The results of BET analysis showed that the average pore diameter of the synthesized hydrogel was $16.7 \mathrm{~nm}$. In general, the size of the pores can be controlled by adjusting the various factors such as the type and amount of surfactant, porosigens and gas forming agent during crosslinking polymerization and the amount of diluent in the monomer mixture (i.e., monomerdiluent ratio). For example, as the amount of diluent (usually water) in the monomer mixture increases, the pore size also increases up to the micrometer $(\mu \mathrm{m})$ range.

The porosity plays the multiple role of enhancing the total water sorption capability and the rate of response by reducing the transport resistance. Therefore, creation of porosity in hydrogels has been considered as an important process in many ways. The phase-separation technique, the water-soluble porogens and the foaming technique are three different methods for preparing porous hydrogel structures. In this paper, as mentioned above, however, the pores were simply produced from water evaporation resulting from reaction medium heat.

Standard calibration curve: The calibration curve of the absorbance as a function of the metronidazole concentration at $245 \mathrm{~nm}$, shown in Fig. 4, has a linear relationship with a correlation coefficient (r) of 0.979 and 0.995 at pHs 1.6 and 7.4 , respectively.
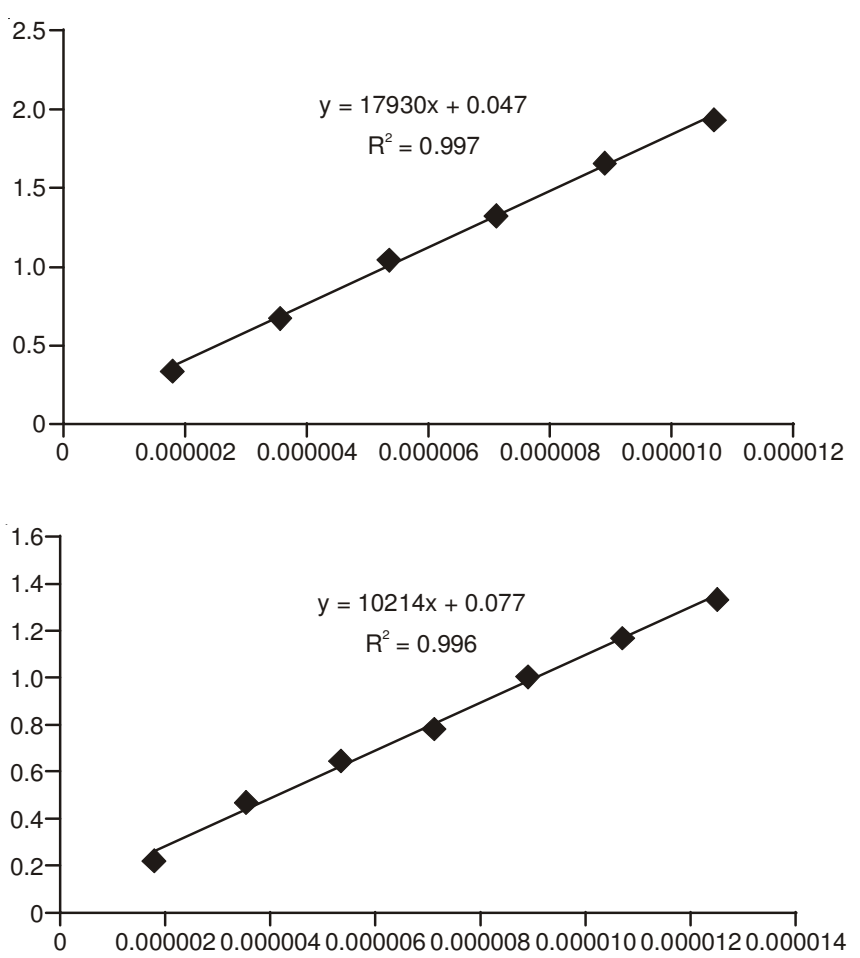

Fig. 4. Standard calibration curve of the absorbance as a function of acetaminophen concentration at $256 \mathrm{~nm}$ on the UV spectrophotometer at $\mathrm{pH} 1.6$ (a) and $\mathrm{pH} 7.4$ (b)

pH-Reversibility for collagen-poly (NaAMPS) hydrogel: Since the hydrogels show different swelling behaviours at various $\mathrm{pHs}$, we investigated their $\mathrm{pH}$-reversibility in the solutions buffered at pHs 1.2 and 7.4 (Fig. 5). The figure shows a stepwise reproducible swelling change of the hydrogel at $25^{\circ} \mathrm{C}$ with alternating $\mathrm{pH}$ between $\mathrm{pHs} 1.2$ and 7.4. At $\mathrm{pH} 7.4$, the hydrogel swells up to $100 \mathrm{~g} / \mathrm{g}$ due to anion-anion repulsive

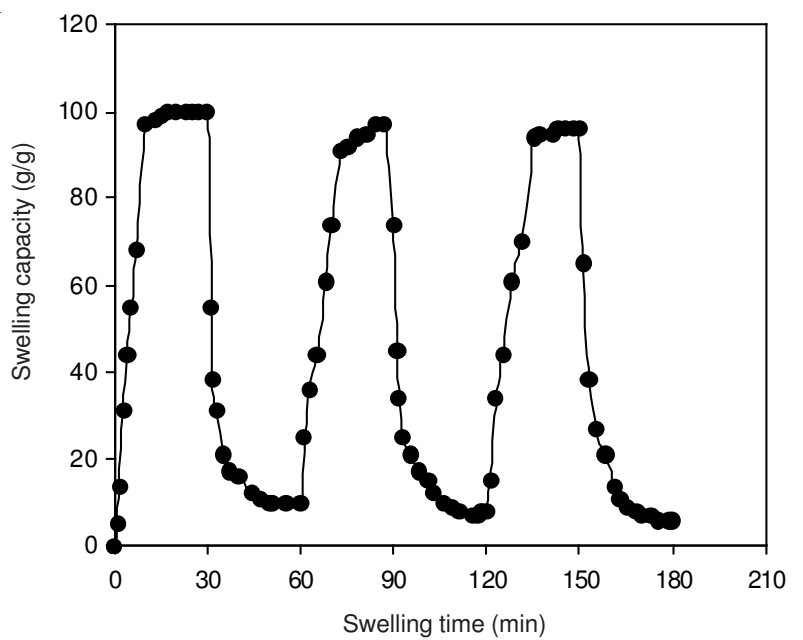

Fig. 5. On-off switching behaviour as reversible pulsatile swelling (pH 7.4) and deswelling ( $\mathrm{pH}$ 1.2) of collagen-g-poly (AMPS) hydrogel

electrostatic forces, while, at $\mathrm{pH} 1.2$, it shrinks within a few minutes due to protonation of carboxylate groups. This sharp swelling-deswelling behaviour of the hydrogels makes them suitable candidates for controlled drug delivery systems. Such on-off switching behaviour as reversible swelling and deswelling we reported for other ionic hydrogels in Fig. 6.

Dry hydrogel
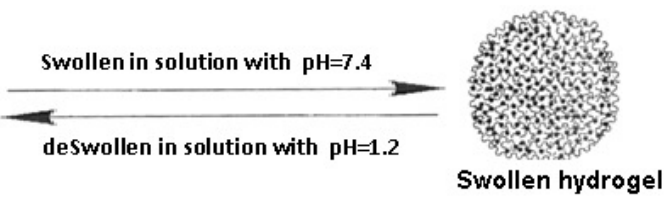

Fig. 6. On-off switching behaviour of hydrogel based on collagen for releasing drug

In vitro cumulative release behaviour of hydrogels: The in vitro release profiles of acetaminophen encapsulated in the polysaccharide-based hydrogels are shown in Fig. 7. The release in simulated gastric fluids (SGF) and simulated intestinal fluids (SIF) displayed $\mathrm{pH}$-sensitivity of these hydrogels. As can be seen from Fig. 5, at acidic $\mathrm{pH}$ value of simulated gastric fluids, the cumulative release ratio of acetaminophen from the hydrogels is below $40 \%$ at the end of the experiment $(24 \mathrm{~h})$, whereas almost $92.5 \%$ of the loaded drug is released within $16 \mathrm{~h}$ in simulated intestinal fluids $(\mathrm{pH} \mathrm{7.4)} \mathrm{medium.} \mathrm{This}$ difference of their swelling behaviour is responsible for the difference of the drug release ratio with changing $\mathrm{pH}$ of the medium. Indeed, the drug in the hydrogel could be released as a result of the hydrogel volume change and interaction between the polymer network and drug. The fractional release is directly proportional to the swelling ratio of the hydrogels. This result indicates that the higher swelling ratios of the hydrogel create larger surface areas to diffuse the drug. In basic solutions ( $\mathrm{pH} 7.4$ ), the electrostatic repulsion between $\mathrm{SO}_{3}{ }^{2-}$ anions of grafted poly (AMPS) on the hydrogel accelerates the release of acetaminophen from the hydrogel.

Fig. 8 shows the schematic of actuation at a distance and resultant squeezing effect for the $\mathrm{pH}$-responsive collagen-based system. Because of the high matrix porosity of the hydrogel, the capillary forces could reinforce the diffusion of solvent into the hydrogel; thereby the acetaminophen release from 


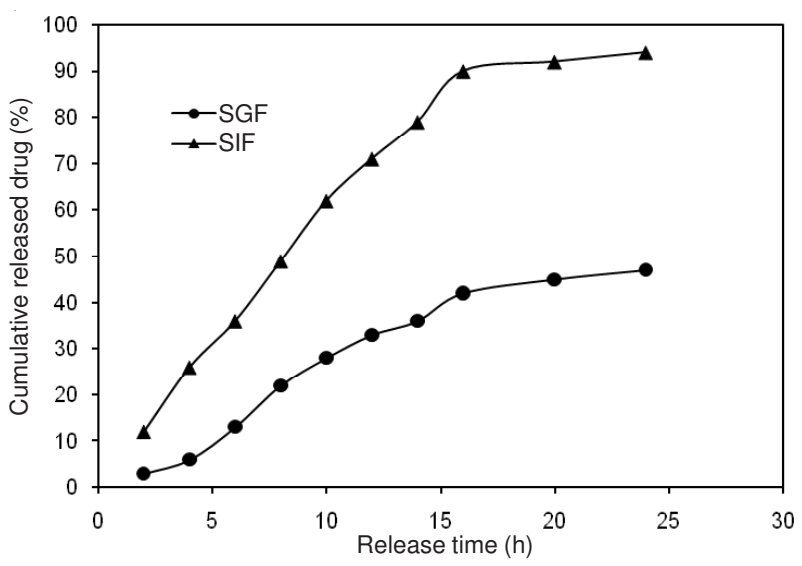

Fig. 7. Release of acetaminophen from hydrogel carrier as a function of $\mathrm{pH}$
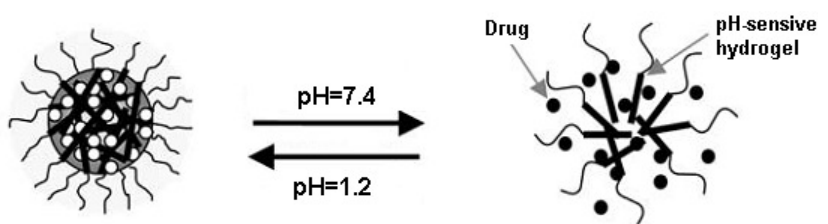

Minimal drug release (Collapsed hydrogel)

Maximal drug relaese (Swollen hydrogel)

Fig. 8. Schematic showing the effect of on-off cycles of $\mathrm{pH}$ on swelling behaviour

the hydrogel matrix occurred mainly due to the diffusion of the drug though the pores of the swelled matrix in the intestinal $\mathrm{pH}$ (7.4).

It shows the $\mathrm{pH}$ triggered collapse and resultant burst release due to squeezing effect.

\section{Conclusion}

A novel protein-based superabsorbent hydrogel was synthesized via graft copolymerization of 2-acrylamido-2methylpropane sulfonic acid onto collagen backbones in an aqueous solution using a persulphate initiator and a hydrophilic crosslinker. The reversible swelling-deswelling behaviour of hydrogel in solutions with acidic and basic $\mathrm{pH}$ makes the hydrogels a suitable candidate for controlled drug delivery systems. So that, the release value of acetaminophen from hydrogels at $\mathrm{pH} 7.4$ was higher than that at $\mathrm{pH} 1.2$ due to the electrostatic repulsion between sulphonate groups. Hence, these results suggest that a porous hydrogel could potentially be a useful local delivery system to release drugs primarily at a specific site of body.

\section{REFERENCES}

1. D.T. Eddington and D.J. Flow, Adv. Drug Deliv. Rev., 56, 199 (2004).

2. M. Hamidi, A. Azadi and P. Rafiei, Adv. Drug Deliv. Rev., 60, 1638 (2008).

3. H. Koo, G. Jin, H. Kang, Y. Lee, H.Y. Nam, H. Jang and G.S. Park, Int. J. Pharm., 374, 58 (2009).

4. S. Kakinoki, T. Taguchi, H. Saito, J. Tanaka and T. Tateishi, Eur. J. Pharm. Biol., 66, 383 (2007).

5. S.J. Kim, G.M. Spinks, S. Prosser, P.G. Whitten, G.G. Wallace and S.I. Kim, Nat. Mater., 5, 48 (2006).

6. H. Kranz and R. Bodmeier, Eur. J. Pharm. Sci., 34, 164 2008).

7. M. Sadeghi and M. Yarahmadi, Orient. J. Chem., 27, 417 (2011).

8. M. Sadeghi and M. Yarahmadi, Orient. J. Chem., 27, 453 (2011).

9. I.C. Kwon, Y.H. Bae and S.W. Kim, Nature, 354, 291 (1991).

10. J.K. Oh, R. Drumright, D.J. Siegwart and K. Matyjaszewski, Prog. Polym. Sci., 33, 448 (2008).

11. J. Siepmann and N.A. Peppas, Adv. Drug Deliv. Rev., 48, 139 (2001). 